\title{
Position Kinematics of a 3-RRS Parallel Manipulator
}

\author{
Halil Tetik ${ }^{*}$, Rohit Kalla ${ }^{\dagger}$, Gokhan Kiper ${ }^{*}$, and Sandipan Bandyopadhyay ${ }^{\dagger}$ \\ * Dept. of Mechanical Engineering, Izmir Institute of Technology, Izmir, Turkey \\ ${ }^{\dagger}$ Dept. of Engineering Design, Indian Institute of Technology Madras, India
}

\begin{abstract}
The 3-RRS parallel manipulator presented in this study comprises of parallel revolute joint axes in each leg. The manipulator is composed of a base and a moving platform which are in the shape of equilateral triangles. Moving platform has two rotational and one translational degrees-of-freedom. This study formulates the forward and inverse kinematics of the parallel manipulator. A $16^{\text {th }}$ order polynomial in terms of one of the passive joint variables is obtained for the forward kinematic analysis. Numerical results and the corresponding pose of the manipulator for inverse and forward kinematics are presented.
\end{abstract}

\section{Introduction}

Parallel manipulators (PM) having less than 6 degrees-of-freedom (DoF) attracted a lot of attention in the past few decades due to their architectural simplicity, low cost and easier control (Fan et al. (2009)). Some of the most successful designs of PMs used in the industry are with less than 6-DoF (Chen et al. (2014)).

This study deals with a 3 -DoF 3 - $\underline{R} R$ S PM which posseses one translational DoF and two rotary ones (Liu and Bonev (2008)). Inverse kinematic analysis of a 3-RRS PM has been performed by (Li et al. (2001)), where the analysis is performed by using a geometrical approach. (Itul and Pisla (2009)) presented the inverse and forward kinematic model for a 3-RRS PM. For the forward position analysis, the set of position equations are suggested to be solved numerically; no analysis as to the number of solutions is presented. In this study, first, the geometry of the 3 - $\underline{R} R S$ PM is described. Then the kinematic constraints are formulated in terms of the loop-closure equations which are solved consequently. Inverse position analysis is done analytically. To solve the forward kinematic problem, the mathematical manipulation proposed by (Srivatsan and Bandyopadhyay (2013)), is applied to a set of three non-linear equations in terms of passive joint variables. Finally, 
a $16^{\text {th }}$ order polynomial in terms of the tangent of the half of one of the passive joint angles is obtained. The formulation is illustrated via numerical examples.

\section{Position Analysis}

The architecture of the PM investigated in this study is presented in Figure 1. The 3-RRS PM consists of a fixed base, a moving platform and

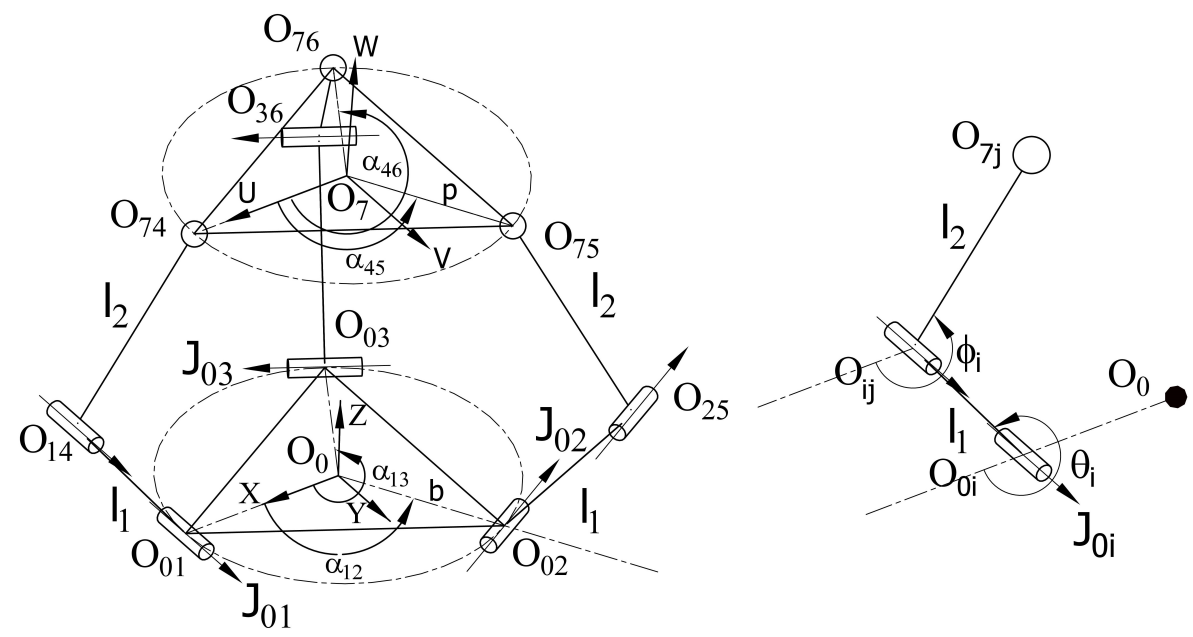

Figure 1. Kinematic model of the 3-RRS PM

three identical limbs. The $i$ th limb is composed of three joints: active revolute (R) joint fixed on the base at the point $O_{0 i}$; passive R joint located at the point $O_{i j}$; and a passive spherical (S) joint between the links having a length $l_{2}$ and the platform, located at the points $O_{7 j}$, for $i=1,2,3$ and $j=4,5,6$.

In Figure 1, a fixed coordinate frame $O_{0}-X Y Z$ is attached to the base. Its origin, $O_{0}$, is chosen as the center of the circle with a radius of $b$, which is the circle tangent to all the three revolute joint axes at the base. The $X$ axis is along the vector $\overrightarrow{O_{0} O_{1}}$ and the $Z$-axis is perpendicular to the base plane. A moving coordinate frame, $O_{7}-U V W$, is attached to the platform. The origin, $O_{7}$, is attached at the center of a circle with a radius $p$, which is the circle passing through the three spherical joint centers. The $U$-axis is along $\overrightarrow{O_{7} O_{74}}$ vector and $W$-axis is perpendicular to the plane of the plat- 
form. In any leg, the axes of the active and passive $\mathrm{R}$ joints are parallel to each other. As a specific case, to achieve a symmetric form, the points $O_{0 i}$ and $O_{7 j}$ are placed at the vertices of the base and moving platform triangles. Therefore, $\alpha_{12}=\angle O_{01} O_{0} O_{02}=120^{\circ}, \alpha_{13}=\angle O_{01} O_{0} O_{03}=240^{\circ}, \alpha_{45}=$ $\angle O_{74} O_{7} O_{75}=120^{\circ}$, and $\alpha_{46}=\angle O_{74} O_{7} O_{76}=240^{\circ}$. The input variables are $\theta_{1}, \theta_{2}$, and $\theta_{3}$; passive revolute joint variables are $\phi_{1}, \phi_{2}$, and $\phi_{3}$.

Constraint Equations. Each limb is constrained to move in plane and the relation in between the $X$ and $Y$ coordinates of the spherical joint centres can be given as $O_{7 j, y}=O_{7 j, x} \tan \left(\alpha_{1 i}\right)$. Constraint equations due to this planar motion are derived by (Tsai (1999)) for a 3-RPS PM and these equation are also valid for the 3 -RRS PM:

$$
\begin{aligned}
& O_{7 y}=-u_{y} p \\
& O_{7 x}=p\left(u_{x}-v_{y}\right) / 2 \\
& u_{y}=v_{x}
\end{aligned}
$$

where $O_{7 x}$ and $O_{7 y}$ are the $X$ and $Y$ coordinates of the platform center, $u_{x}, u_{y}, v_{x}, v_{y}$ are the corresponding elements of the rotation matrix and $p$ is the radius of the circle attached to the moving platform.

$\mathrm{O}_{7 z}$ remains independent, indicating the translational DoF along the Z-axis. To determine the elements of the rotation matrix $[\mathbf{R}]$ between coordinate frames of the base and moving platform, the following rotation sequence is applied: first rotate about the fixed $Z$-axis by an angle of $\psi_{z}$, then about the fixed $Y$-axis by an angle of $\psi_{y}$ and finally about the fixed $X$-axis by an angle of $\psi_{x}$. This results in:

$$
[\mathbf{R}]=\left[\begin{array}{lll}
u_{x} & v_{x} & w_{x} \\
u_{y} & v_{y} & w_{y} \\
u_{z} & v_{z} & w_{z}
\end{array}\right]=\left[\begin{array}{ccc}
\mathrm{c}_{y} c_{z} & -\mathrm{c}_{y} \mathrm{~s}_{z} & \mathrm{~s}_{y} \\
s_{x} \mathrm{~s}_{y} \mathrm{c}_{z}+c_{x} \mathrm{~s}_{z} & \mathrm{c}_{x} c_{z}-s_{x} s_{y} \mathrm{~s}_{z} & -\mathrm{s}_{x} \mathrm{c}_{y} \\
\mathrm{~s}_{x} \mathrm{~s}_{z}-\mathrm{c}_{x} \mathrm{~s}_{y} \mathrm{c}_{z} & \mathrm{~s}_{x} c_{z}+c_{x} s_{y} s_{z} & \mathrm{c}_{x} \mathrm{c}_{y}
\end{array}\right]
$$

where s and $\mathrm{c}$ stand for sin and cos, respectively, and subscripts $x, y$ and $z$ stand for rotation angles $\psi_{x}, \psi_{y}$ and $\psi_{z}$, respectively. $[\mathbf{R}]$ is bound to the 6 orthogonality conditions for rotation matrices. Besides, Eq. (3) further constrains $[\mathbf{R}]$. Therefore, only two elements of $[\mathbf{R}]$ are independent. Considering the workspace of the 3 - $\underline{R} R S$ PM, the suitable choices of independent parameters are $w_{x}$ and $w_{y}$, while $O_{7 z}, w_{x}$ and $w_{y}$ are the independent workspace parameters. For given $w_{x}$ and $w_{y}$, first $\psi_{x}, \psi_{y}$ and $\psi_{z}$ can be determined:

$$
\begin{aligned}
& \psi_{y}=\sin ^{-1}\left(w_{x}\right), \text { where, } s_{y} \neq \pm 1 \\
& \psi_{x}=\sin ^{-1}\left(-w_{y} / c_{y}\right)
\end{aligned}
$$


Imposing Eq. (3) in Eq. (4), and the dividing by $c_{z}$, we can determine $\psi_{z}$ as

$$
\psi_{z}=\tan ^{-1}\left(-\mathrm{s}_{x} \mathrm{~s}_{y} /\left(\mathrm{c}_{x}+\mathrm{c}_{y}\right)\right)
$$

Substituting $\psi_{x}, \psi_{y}$ and $\psi_{z}$ given by Eqs. (5)-(7), [R] can be determined from Eq. (4). Eqs. (1)-(2) give the dependent position parameters.

Inverse Position Analysis. Inverse kinematics problem is to find the input angles $\theta_{1}, \theta_{2}$ and $\theta_{3}$ for a given pose of the moving platform. The loop-closure equation can be written as:

$$
\begin{aligned}
& \overrightarrow{O_{0} O_{7 j}}=\left[\mathbf{R}_{Z}\left(\alpha_{1 i}\right)\right]\left\{\left[\begin{array}{l}
b \\
0 \\
0
\end{array}\right]+\left[\mathbf{R}_{Y}\left(\theta_{i}\right)\right]\left[\begin{array}{c}
l_{1} \\
0 \\
0
\end{array}\right]+\left[\mathbf{R}_{Y}\left(\phi_{i}\right)\right]\left[\begin{array}{c}
l_{2} \\
0 \\
0
\end{array}\right]\right\} \\
& \overrightarrow{O_{0} O_{7 j}}=\overrightarrow{O_{0} O_{7}}+[\mathbf{R}] \cdot\left[\mathbf{R}_{Z}\left(\alpha_{4 j}\right)\right]\left(\begin{array}{l}
p \\
0 \\
0
\end{array}\right)
\end{aligned}
$$

for $i=1,2,3$ and $j=i+3$. [ $\left.\mathbf{R}_{Y}\right]$ and $\left[\mathbf{R}_{Z}\right]$ are the elementary CCW rotation matrices about $Y$-, and $Z$-axes, respectively. We express the points $O_{7 j}$ in terms of the given pose parameters using Eq. (8). The $X$ and $Z$ components of Eq. (8) are:

$$
\begin{aligned}
& l_{2} c_{\phi i} c_{\alpha 1 i}=O_{7 j x}-c_{\alpha 1 i}\left(b+l_{1} c_{\theta i}\right) \\
& l_{2} s_{\phi i}=-O_{7 j z}-l_{1} s_{\theta i}
\end{aligned}
$$

Solving $c_{\phi i}$ and $s_{\phi i}$ from the Eqs. (9)-(10), and using the identity $c_{\phi i}^{2}+$ $s_{\phi}^{2}=1$ :

$$
\begin{aligned}
& l_{2}^{2}\left(c_{\alpha 1 i}+1\right)^{2}-\left[O_{7 j x} c_{\alpha 1 i}\left(b+l_{1} c_{\theta i}\right)\right]^{2}-\left[c_{\alpha 1 i}\left(O_{7 j z} l_{1} s_{\theta i}\right)\right]^{2}=0 \\
& \Rightarrow A_{i} c_{\theta i}+B_{i} s_{\theta i}+C_{i}=0
\end{aligned}
$$

where $A_{i}=2 l_{1} c_{\alpha 1 i}\left(-O_{7 j x}+b c_{\alpha 1 i}\right), B_{i}=2 l_{1} O_{7 j z} c_{\alpha 1 i}^{2}$ and $C_{i}=O_{7 j x}^{2}-$ $2 b O_{7 j x} c_{\alpha 1 i}+c_{\alpha 1 i}^{2}\left(b^{2}+l_{1}^{2}-l_{2}^{2}+O_{7 j z}^{2}\right)$. Applying tangent of half angle substitution to Eq. (11) and solving for $\theta_{i}$ :

$$
\theta_{i}=2 \operatorname{atan} 2\left(-B_{i} \pm \sqrt{A_{i}^{2}+B_{i}^{2}-C_{i}^{2}}, C_{i}-A_{i}\right)
$$


$A_{i}=C_{i}$ results in singularity, which is not considered in this paper and it is assumed that $A_{i} \neq C_{i}$. Due to the \pm sign in Eq. (12), each $\theta_{i}$ has two possible values, which yields at most 8 solutions. Therefore the PM has 8 assembly modes for the inverse kinematics.

Forward Position Analysis. The coordinates of the points $O_{7 j}$ can be calculated in terms of input variables $\theta_{i}$ and passive $\mathrm{R}$ joint variables $\phi_{i}$ by making use of the Eq. (8). Since the moving platform is in the form of an equilateral triangle, the distances between $O_{7 j}$ are constant and can be calculated by the cosine theorem. Using Eq. (8):

$$
\begin{aligned}
& 3 p^{2}=d^{2}=\left|O_{74} O_{75}\right|^{2} \Rightarrow f_{1}\left(\phi_{1}, \phi_{2}\right)=0 \\
& 3 p^{2}=d^{2}=\left|O_{75} O_{76}\right|^{2} \Rightarrow f_{2}\left(\phi_{2}, \phi_{3}\right)=0 \\
& 3 p^{2}=d^{2}=\left|O_{76} O_{74}\right|^{2} \Rightarrow f_{3}\left(\phi_{1}, \phi_{3}\right)=0
\end{aligned}
$$

Eqs. (13)-(14) can be rewritten as:

$$
\begin{aligned}
& f_{10}+f_{11} \mathrm{~s} \phi_{2}+f_{12} \mathrm{c} \phi_{2}=0 \\
& f_{20}+f_{21} \mathrm{~s} \phi_{2}+f_{22} \mathrm{c} \phi_{2}=0
\end{aligned}
$$

Solving for $c \phi_{2}, s \phi_{2}$ from there,

$$
\mathrm{s} \phi_{2}=\frac{f_{12} f_{20}-f_{10} f_{22}}{f_{11} f_{22}-f_{12} f_{21}}, \mathrm{c} \phi_{2}=\frac{f_{10} f_{21}-f_{11} f_{20}}{f_{11} f_{22}-f_{12} f_{21}}
$$

Since, $c^{2} \phi_{2}+s^{2} \phi_{2}=1$, from Eq. (16)-(17), assuming $f_{11} f_{22}-f_{12} f_{21} \neq 0$ :

$$
\left(f_{12} f_{20}-f_{10} f_{22}\right)^{2}+\left(f_{10} f_{21}-f_{11} f_{20}\right)^{2}-\left(f_{11} f_{22}-f_{12} f_{21}\right)^{2}=0
$$

At this point we apply the tangent of half angle substitution for the angles $\phi_{1}$ and $\phi_{3}$ for Eqs. (13)-(15): $t_{1}=\tan \left(\phi_{1} / 2\right)$ and $t_{3}=\tan \left(\phi_{3} / 2\right)$. Eq. (17) involves $t_{1}$ and $t_{3}$, only. Rearranging Eq. (17), it is seen that it is a $4^{\text {th }}$ degree polynomial in $t_{1}$ :

$$
a_{0}+a_{1} t_{1}+a_{2} t_{1}^{2}+a_{3} t_{1}^{3}+a_{4} t_{1}^{4}=0
$$

Coefficients $a_{i}$ in Eq. (20) are also $4^{\text {th }}$ degree polynomials in $t_{3}$. Also rearranging Eq. (15) in terms of $t_{1}$ results in a quadratic equation:

$$
b_{0}+b_{1} t_{1}+b_{2} t_{1}^{2}=0
$$

The coefficients $b_{i}$ are also $2^{\text {nd }}$ degree polynomials in terms of $t_{3}$. Eliminating $t_{1}$ from Eqs. (20)-(21) using polynomial division, we obtain:

$$
\frac{b_{2}^{3} H}{\left[a_{4} b_{1}^{3}-b_{1}\left(2 a_{4} b_{0}+a_{3} b_{1}\right) b_{2}+\left(a_{3} b_{0}+a_{2} b_{1}\right) b_{2}^{2}-a_{1} b_{2}^{3}\right]^{2}}=0 \Rightarrow H=0
$$


where

$$
\begin{aligned}
H= & a_{4}\left\{a_{4} b_{0}^{4}+b_{1}\left[-a_{3} b_{0}^{3}+b_{1}\left(a_{2} b_{0}^{2}-a_{1} b_{0} b_{1}+a_{0} b_{1}^{2}\right)\right]\right\}+b_{2}\left(a_{3}^{2}-2 a_{2} a_{4}\right) b_{0}^{3}+ \\
& b_{2}^{2}\left[\left(a_{2}^{2}-2 a_{1} a_{3}+2 a_{0} a_{4}\right) b_{0}^{2}+\left(-a_{1} a_{2}+3 a_{0} a_{3}\right) b_{0} b_{1}+a_{0} a_{2} b_{1}^{2}\right]+ \\
& \left(-a_{2} a_{3}+3 a_{1} a_{4}\right) b_{0}^{2} b_{1}+\left(a_{1} a_{3}-4 a_{0} a_{4}\right) b_{0} b_{1}^{2}-a_{0} a_{3} b_{1}^{3}+ \\
& b_{2}^{3}\left[\left(a_{1}^{2}-2 a_{0} a_{2}\right) b_{0}-a_{0} a_{1} b_{1}\right]+a_{0}^{2} b_{2}^{4}
\end{aligned}
$$

Eq. (22) is valid provided that $b_{2} \neq 0$ and also denominator in Eq. (22) is nonzero. Eq. (22) is a $16^{\text {th }}$ order polynomial in terms of $t_{3}$. The value of $t_{3}$ can be numerically computed from Eq. (22). For any $t_{3}$ value, $t_{1}$ is determined from Eqs. (20)-(21) and $\phi_{2}$ is given by Eq. (16). There are at most 16 assembly modes of the moving platform for the forward kinematics. Once the platform points $O_{7 j}$ are determined from Eq. (8), the location of the platform is determined.

\section{$3 \quad$ Numerical Example}

Several numerical examples are worked out in order to check forward and inverse kinematic solutions using Mathematica. As an example, consider the case where $b=0.55 \mathrm{~m}, p=0.275 \mathrm{~m}, l_{1}=0.7 \mathrm{~m}$ and $l_{2}=0.775 \mathrm{~m}$. For the inverse kinematic analysis, as a generic example for given pose parameters $O_{7 z}=1.2 \mathrm{~m}, w_{x}=-0.2$ and $w_{y}=0.2$, the possible corresponding joint angles are found as $\left(\theta_{1}, \theta_{2}, \theta_{3}\right)=\left(-71.60^{\circ},-66.09^{\circ},-68.57^{\circ}\right)$, $\left(-133.61^{\circ},-144.85^{\circ},-136.47^{\circ}\right)$. Any of two solutions for an angle is valid and there are totally eight solutions.

To verify the forward kinematic formulation, let $\theta_{1}=-133.61^{\circ}, \theta_{2}=$ $-144.85^{\circ}$ and $\theta_{3}=-136.47^{\circ}$, which is one of the eight solutions above. The solution of the $16^{\text {th }}$ order univariate polynomial in terms of $t_{3}$ results in sixteen distinct real solutions. The numerical values obtained for $t_{3}$, corresponding passive joint variables $\phi_{i}$ and task space variables $O_{7 z}, w_{x}$ and $w_{y}$ are presented in Figure 2. Notice that the $6^{\text {th }}$ set of solutions matches with the task space parameters used in the inverse kinematic analysis. Figure 2 depicts the poses of the PM for the corresponding solutions.

\section{Conclusion}

A detailed analysis for the forward and inverse kinematics analysis of the 3$\underline{R R S}$ PM is presented in this study. For the forward kinematics, all but one dependent joint variables are eliminated to obtain a $16^{\text {th }}$ order polynomial. This suggests that an upper bound for the forward kinematics solutions of the 3 - $\underline{R} R S$ PM is 16, which proves that polynomial obtained is the smallest 


\begin{tabular}{|l|l|l|l|l|}
\hline & \\
\hline $\mathrm{t}_{3}=-2.07$ \\
$\phi_{1}=-56.04^{\circ}$ \\
$\phi_{2}=-92.32^{\circ}$ \\
$\phi_{3}=-128.40^{\circ}$ \\
$\mathrm{w}_{\mathrm{x}}=-0.034$ \\
$\mathrm{w}_{\mathrm{y}}=-0.18$ \\
$\mathrm{O}_{7, \mathrm{z}}=1.14$
\end{tabular}

Figure 2. Forward kinematic solutions

one. The formulations are implemented in Mathematica and several numerical examples are presented. Indeed 16 real solutions are obtained for some configurations.

The formulations developed here will be implemented for the control of a 3-RRS PM in Izmir Institute of Technology. Further studies would include the identification of singularities and safe working zone, (see, Srivatsan and Bandyopadhyay (2014)) and dynamic analyses of the PM. 


\section{Bibliography}

Xiang Chen, FG Xie, XJ Liu, F Xie, and T Sun. A comparison study on motion/force transmissibility of two typical 3-DoF parallel manipulators: the sprint Z3 and A3 tool heads. International Journal of Advanced Robotic System, 11(5):1-10, 2014.

Caixia Fan, Hongzhao Liu, and Yarbin Zhang. Kinematics and singularity analysis of a novel 1T2R fully-decoupled parallel mechanism. In Intelligent Computing and Intelligent Systems, 2009. ICIS 2009. IEEE International Conference, volume 2, pages 312-316. IEEE, 2009.

Tiberiu Itul and Doina Pisla. Kinematics and dynamics of 3-DoF parallel robots with triangle platform. Journal of Vibroengineering, 11(1), 2009.

Jianfeng Li, Jinsong Wang, Wusheng Chou, Yuru Zhang, Tianmiao Wang, and Qixian Zhang. Inverse kinematics and dynamics of the 3-RRS parallel platform. In Robotics and Automation, 2001. Proceedings 2001 ICRA. IEEE International Conference, volume 3, pages 2506-2511. IEEE, 2001.

Xin-Jun Liu and Ilian A Bonev. Orientation capability, error analysis, and dimensional optimization of two articulated tool heads with parallel kinematics. Journal of Manufacturing Science and Engineering, 130(1): 011015-011015-9, 2008.

$\mathrm{R}$ Arun Srivatsan and Sandipan Bandyopadhyay. On the position kinematic analysis of MaPaMan: A reconfigurable three-degrees-of-freedom spatial parallel manipulator. Mechanism and Machine Theory, 62:150165, 2013.

R Arun Srivatsan and Sandipan Bandyopadhyay. Determination of the Safe Working Zone of a Parallel Manipulator. In Computational Kinematics, pages 201-208. Springer, 2014.

Lung-Wen Tsai. Robot analysis: The Mechanics of Serial and Parallel Manipulators. John Wiley \& Sons, 1999. 